\title{
Optical probe of ferroelectric order in bulk and thin-film perovskite titanates
}

\author{
M. Rössle, ${ }^{1, *}$ C. N. Wang, ${ }^{1}$ P. Marsik, ${ }^{1}$ M. Yazdi-Rizi, ${ }^{1}$ K. W. Kim,,${ }^{1,2}$ A. Dubroka, ${ }^{1,3}$ I. Marozau, ${ }^{1}$ C. W. Schneider, ${ }^{4}$ \\ J. Humlíček, ${ }^{3}$ D. Baeriswyl, ${ }^{1}$ and C. Bernhard ${ }^{1, \dagger}$ \\ ${ }^{1}$ University of Fribourg, Department of Physics and Fribourg Center for Nanomaterials, Chemin du Musée 3, CH-1700 Fribourg, Switzerland \\ ${ }^{2}$ Department of Physics, Chungbuk National University, Cheongju 361-763, Korea \\ ${ }^{3}$ Department of Condensed Matter Physics, Faculty of Science, Masaryk University and Central European Institute of Technology, \\ Kotlářská 2, CZ-61137 Brno, Czech Republic \\ ${ }^{4}$ Paul Scherrer Institut, $\mathrm{CH}-5232$ Villigen, Switzerland
}

(Received 3 June 2013; revised manuscript received 22 August 2013; published 26 September 2013)

\begin{abstract}
We have measured the temperature dependence of the direct band gap $E_{g}$ in $\mathrm{SrTiO}_{3}$ and $\mathrm{BaTiO}_{3}$ and related materials with quantum-paraelectric and ferroelectric properties using optical spectroscopy. We show that $E_{g}$ exhibits an anomalous temperature dependence with pronounced changes in the vicinity of the ferroelectric transition that can be accounted for in terms of the Fröhlich electron-phonon interaction with an optical phonon mode, the so-called soft mode. In addition, we demonstrate that these characteristic changes of $E_{g}$ can be readily detected even in very thin films of $\mathrm{SrTiO}_{3}$ with a strain-induced ferroelectric order. Optical spectroscopy thus can be used as a rather sensitive probe of ferroelectric order in very thin films of these titanates and probably also in subsequent multilayers and devices.
\end{abstract}

DOI: 10.1103/PhysRevB.88.104110

PACS number(s): 77.80.-e, 63.20.kd, 77.84.Cg, 78.20.-e

\section{INTRODUCTION}

Ferroelectric (FE) materials have important applications in sensors and electronic devices, for example, in dynamic random access memories. ${ }^{1-3}$ The growth and characterization of very thin FE layers is therefore an active field of research. In FE thin films, the polarization and the transition temperature $T_{\text {Curie }}$ can be severely reduced below their bulk values or even entirely suppressed due to so-called "dead layers" that may arise from poorly screened depolarization fields at surfaces and interfaces or from strain and defects. ${ }^{1-3}$ On the other hand, in $\mathrm{SrTi}^{16} \mathrm{O}_{3}$, which in its bulk form is a so-called quantum-paraelectric material for which the $\mathrm{FE}$ order is suppressed by quantum lattice fluctuations, a FE order can be induced in thin films that are strained by lattice matching to the substrate. ${ }^{4,5}$ For such thin films and especially for subsequent complex heterostructures with FE layers, it is therefore very important to have a readily accessible technique that enables one to identify the FE order. Direct capacitance or impedance spectroscopy measurements of the FE order on such thin films are often complicated and hindered by parasitic charges forming at interfaces and electrodes that can obscure the FE contribution. ${ }^{6,7}$ Dedicated techniques such as piezoresponse force microscopy, ${ }^{8}$ synchrotron X-ray diffraction, ${ }^{9,10}$ or ultraviolet Raman spectroscopy ${ }^{11}$ have therefore been used to detect the FE order in very thin films. In the following, we show that standard optical spectroscopy measurements of the temperature $(T)$ dependence of the direct band gap $E_{g}$ can be used as an alternative and very effective tool to identify such a FE transition. This is demonstrated for the case of bulk $\mathrm{SrTi}^{16} \mathrm{O}_{3}$ and $\mathrm{BaTiO}_{3}$ crystals as well as for strained $\mathrm{SrTi}^{16} \mathrm{O}_{3}$ thin films.

\section{EXPERIMENTAL DETAILS}

$\mathrm{SrTi}^{16} \mathrm{O}_{3}$ (STO) and $\mathrm{KTaO}_{3}$ crystals were purchased from Crystec ${ }^{12}$ and a $\mathrm{BaTiO}_{3}$ crystal from SurfaceNet, ${ }^{13}$ respectively. A polycrystalline $\mathrm{CaTiO}_{3}$ sample was made via solid-state sintering. A ${ }^{18} \mathrm{O}$ exchanged $\mathrm{SrTi}^{18} \mathrm{O}_{3}$ crystal was prepared as described in Ref. 14. STO thin films on $\mathrm{DyScO}_{3}$ and $\left(\mathrm{LaAlO}_{3}\right)_{0.3}-\left(\mathrm{SrAlTaO}_{6}\right)_{0.7}$ (LSAT) substrates were prepared by pulsed laser deposition with in situ reflection high-energy electron diffraction growth control. The substrate temperature was $900{ }^{\circ} \mathrm{C}$, the oxygen pressure $p\left(\mathrm{O}_{2}\right)=$ $0.11 \mathrm{mbar}$, and we used a $248-\mathrm{nm}$ excimer laser with a fluence of $1.5 \mathrm{~J} / \mathrm{cm}^{2}$. Subsequent to the growth, the films were slowly cooled in 1-bar oxygen.

Spectroscopic ellipsometry measurements were performed in the near-infrared to ultraviolet range of $0.5-6.5 \mathrm{eV}$ with a commercial ellipsometer (WOOLLAM VASE ${ }^{15}$ ) equipped with a UHV cryostat for a temperature range of $4 \mathrm{~K}<T \leqslant$ $700 \mathrm{~K}$. For the far-infrared (FIR) and mid-infrared measurements, we used a home-built setup as described in Ref. 16. FIR reflectivity measurements were performed with the home-built setup described in Ref. 17. The modeling of the ellipsometry data (surface roughness correction for crystals and substrate correction for thin films) was done with the WOOLLAM VASE software. ${ }^{15}$

\section{RESULTS AND DISCUSSION}

Figure 1(a) shows the $T$-dependent spectra from $2-6 \mathrm{eV}$ of the real and imaginary parts of the dielectric function $\varepsilon=$ $\varepsilon_{1}+\mathrm{i} \varepsilon_{2}$ of bulk STO. They contain four prominent bands centered at 3.7, 4.2, 5.1, and about $6 \mathrm{eV}$ which correspond to direct interband transitions between the O- $2 p$ states in the valence band and the Ti-3 $d$ states in the conduction band. ${ }^{18,19}$ The indirect band gap at $3.25 \mathrm{eV}$ yields a very weak absorption band that is barely visible in the spectra and therefore does not concern the following discussion of the $T$ dependence of the direct band gap $E_{g}$ at about $3.7 \mathrm{eV}$. Figure 1(b) shows the spectra of the second derivative of $\varepsilon_{2}$ as obtained with a Savitzky-Golay smoothing procedure. ${ }^{20}$ They have been fitted with the second derivative of the function

$$
\varepsilon(\omega)=B-S e^{i 3 \pi / 2}(\omega-E+i \Gamma)^{1 / 2},
$$



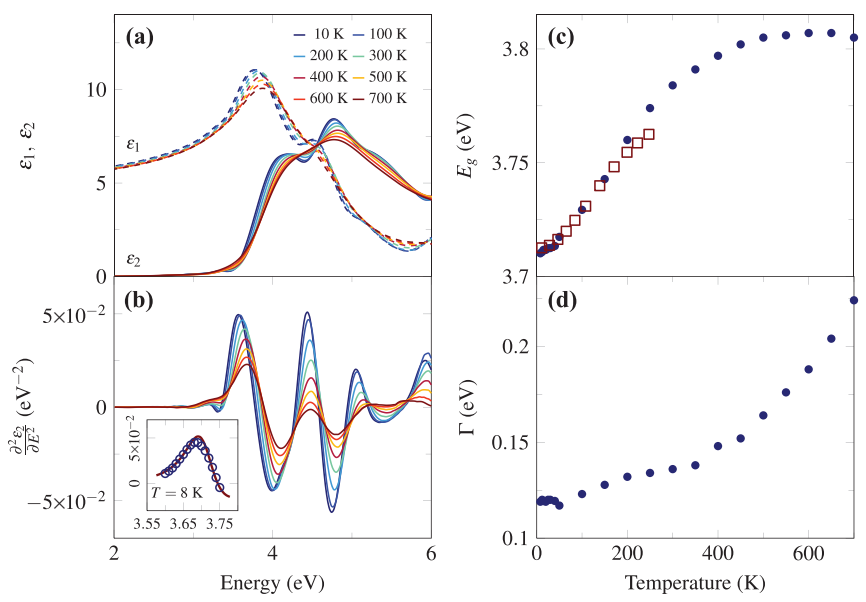

FIG. 1. (Color online) (a) $T$-dependent spectra of the dielectric function $\varepsilon=\varepsilon_{1}+\mathrm{i} \varepsilon_{2}$ of STO. (b) Corresponding second derivative spectra of $\varepsilon_{2}$. $T$ dependence of (c) the energy $E_{g}$ and (d) the broadening $\Gamma$ of the direct gap (full circles) as obtained by fitting with the second derivative of Eq. (1). Open squares in (c) show the contribution of the Fröhlich electron-phonon interaction to the $T$ dependence of $E_{g}$ below $250 \mathrm{~K}$ as estimated with Eq. (2). The inset in (b) shows a representative fit of the lowest direct interband transition (solid line) using Eq. (1) to the second derivative of the experimental data (open symbols) at $T=8 \mathrm{~K}$.

which describes the response in the vicinity of a direct absorption edge due to the singularity in the joint density of states at a three-dimensional so-called $M_{0}$ critical point. ${ }^{21,22}$ Further details are given in Appendix A. Here, the parameter $B$ represents a constant background, $S$ is the amplitude, $\Gamma$ the broadening, and $E$ corresponds to the energy threshold of the associated transition which in this case is the energy of the band gap $E_{g}$. The obtained $T$ dependence of $E_{g}$ and $\Gamma$ is displayed in Figs. 1(c) and 1(d), respectively. The evolution of $E_{g}$ is rather anomalous; it exhibits a sizable increase from $\sim 3.71 \mathrm{eV}$ at $10 \mathrm{~K}$ to $3.77 \mathrm{eV}$ at $300 \mathrm{~K}$ before it reaches a maximum at $\sim 3.81 \mathrm{eV}$ around $500 \mathrm{~K}$ and decreases again toward higher $T$. We note that this characteristic $T$-dependent shift of the interband transition is visible in the bare spectra of $\varepsilon$, i.e., it can be readily identified even without the quantitative analysis using Eq. (1). A similar trend below $300 \mathrm{~K}$ was also previously reported in Ref. 23.

The magnitude of $E_{g}$ in semiconductors and insulators is well known to undergo sizable changes with $T$, usually to lower energy, as in $\mathrm{Si}, \mathrm{Ge}$, or GaAs, ${ }^{24,25}$ but in some cases as in $\mathrm{PbS}$ also to higher energy. ${ }^{26}$ Part of this $T$ dependence is accounted for by the thermal expansion of the lattice, but a sizable contribution can also arise from the electron-phonon interaction. ${ }^{27,28}$ The latter is caused by the deformation of the electronic potentials due to the dynamic atomic displacements. Its sign is determined by the lattice structure and the electronic states forming the valence and conduction bands. Its magnitude depends on the amplitude of the atomic displacement $u$, which in the harmonic approximation is related to the effective mass of the atoms $\mu$, the eigenfrequency of the phonon mode $\omega$, and the Bose-Einstein factor $n_{B}=\left(e^{\hbar \omega / k_{B} T}-1\right)^{-1}$, according to $\left\langle u^{2}\right\rangle=\frac{\hbar}{2 \mu \omega}\left(1+2 n_{B}\right)$ where $\langle\cdots\rangle$ means a thermal average. The resulting shift of the band gap $\Delta E_{g}$ is nearly constant at $k_{B} T \ll \hbar \omega$ where it is dominated by the quantum lattice fluctuations, it starts to increase around $k_{B} T \approx \hbar \omega$, and is proportional to $T$ at $k_{B} T \gg \hbar \omega .{ }^{29}$ As observed in some chalcopyrites, the $T$ dependence of $E_{g}$ may also exhibit a sign change if the contributions due to phonons with low and high eigenfrequencies have opposite signs. ${ }^{30}$

In polar or partially ionic crystals, an additional contribution to the electron-phonon interaction arises from the long wavelength longitudinal optical (LO) phonons which give rise to a macroscopic polarization that can be described in terms of the Fröhlich interaction. ${ }^{21}$ Its contribution to the shift of the band gap is ${ }^{27,31}$

$$
\Delta E_{g}=-A\left(\varepsilon_{\infty}^{-1}-\varepsilon_{0}^{-1}\right)\left(1+2 n_{B}\right),
$$

where $\varepsilon_{\infty}$ and $\varepsilon_{0}$ are the dielectric constants at energies well above and below the phonon range, respectively, and $A$ is a $T$-independent prefactor that depends on material parameters such as effective mass and lattice constant. It is important to notice that at $k_{B} T \ll \hbar \omega$, where $n_{B}$ is nearly constant, the $T$ dependence of $\Delta E_{g}$ is determined by that of $\varepsilon_{0}$.

In the following, we provide evidence that in STO and related titanates this Fröhlich interaction governs the $T$ dependence of $E_{g}$ below about $250 \mathrm{~K}$. In STO, the thermal expansion of the lattice has a regular $T$ dependence ${ }^{32,33}$ and thus should contribute to a decrease of $E_{g}$ as the lattice expands with increasing $T$. This assumption is confirmed by band-structure calculations which predict that $E_{g}$ increases with pressure (or decreasing lattice constant). ${ }^{34}$ The structural phase transitions at $105 \mathrm{~K}$ from a cubic to a tetragonal state and around $65 \mathrm{~K}$ toward an orthorhombic phase also do not have any noticeable effect on $E_{g}$. This suggests that the unusual $T$ dependence of $E_{g}$ in STO is caused by the electron-phonon interaction. STO is indeed well known for its anomalous lattice dynamical properties. It is a so-called quantum-paraelectric material with an incipient FE state below about $35 \mathrm{~K}$ (Ref. 35) that is suppressed by the quantum lattice fluctuations. ${ }^{36}$ This anomalous behavior is closely related to the softening of a transverse optical (TO) phonon which involves a polar displacement of the Ti ion away from the central position of the octahedron formed by the neighboring oxygen ions. The eigenfrequency of this so-called "soft mode" $\omega_{\mathrm{TO}}$ decreases from about $95 \mathrm{~cm}^{-1}$ at $300 \mathrm{~K}$ to about $15 \mathrm{~cm}^{-1}$ at low $T .{ }^{37}$ We have also measured the $T$ dependence of $\omega_{\mathrm{TO}}$ with far-infrared ellipsometry as described in Appendix B and shown in Fig. 4(a). According to the Lyddane-Sachs-Teller relation $\frac{\varepsilon_{0}}{\varepsilon_{\infty}}=C \frac{\omega_{\mathrm{LO}}^{2}}{\omega_{\mathrm{TO}}^{2}}$, this soft TO mode gives rise to a strong increase of $\varepsilon_{0}=390$ at $300 \mathrm{~K}$ to $\varepsilon_{0}=14.000$ at low $T .^{38,39} \mathrm{The}$ open squares in Fig. 1(c) show that this divergence of $\varepsilon_{0}$ toward low $T$ and the subsequent increase of the Fröhlich interaction according to Eq. (2) account well for the renormalization of $E_{g}$ below about $250 \mathrm{~K}$. The fit to the $E_{g}$ data yields a vertical offset of $E_{g}(T=0 \mathrm{~K})=4.15 \pm 0.02 \mathrm{eV}$ and a prefactor of $A=2.26 \pm 0.12 \mathrm{eV}$. The prefactor $C=2.52 \pm 0.12$ in the Lyddane-Sachs-Teller relation, which accounts for the $T$-independent contribution of the two other IR-active phonon modes at 175 and $540 \mathrm{~cm}^{-1}$, and the values of $\varepsilon_{\infty}=5.1$ and the eigenfrequency of the LO mode $\omega_{\mathrm{LO}}=788 \mathrm{~cm}^{-1}$, have been obtained from the ellipsometry data as is described in Appendixes $\mathrm{C}$ and $\mathrm{D}$, respectively. The latter have only a very 

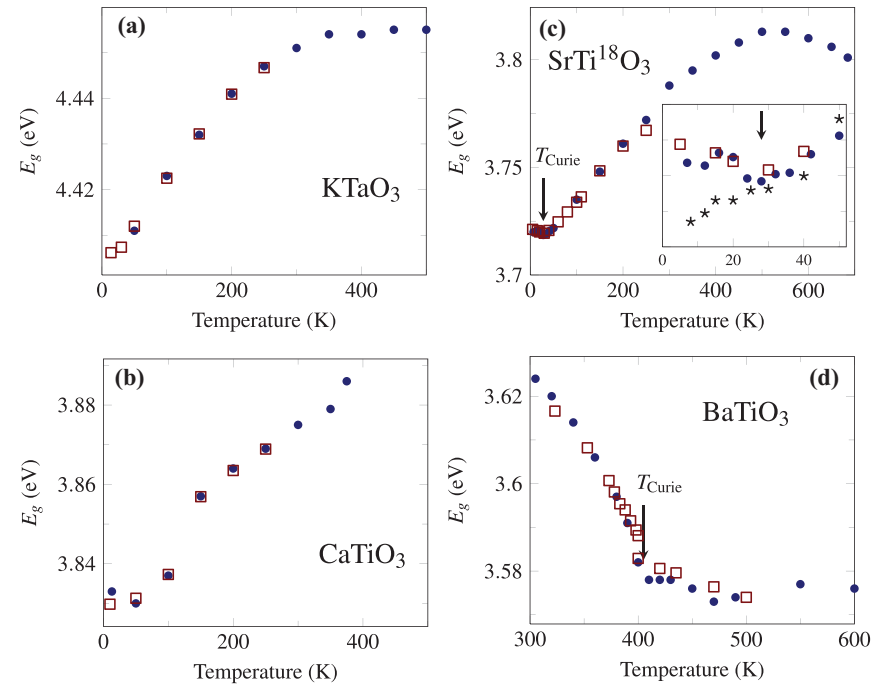

FIG. 2. (Color online) $T$ dependence of $E_{g}$ (full circles) in (a) $\mathrm{KTaO}_{3}$ and (b) $\mathrm{CaTiO}_{3}$. Open squares show the contribution of the Fröhlich interaction as obtained with Eq. (2). (c) Corresponding data (full circles) and calculations (open squares) for $\mathrm{SrTi}^{18} \mathrm{O}_{3}$ with a FE transition at $T_{\text {Curie }}=26 \mathrm{~K}$ as marked by the arrow. Inset: Magnification of the low- $T$ regime with the rescaled data of STO (stars) shown for comparison. (d) Corresponding data and calculations for $\mathrm{FE} \mathrm{BaTiO}_{3}$ with $T_{\text {Curie }}=405 \mathrm{~K}$.

weak $T$ dependence as shown (see Figs. 5 and 6) that does not strongly influence our estimates for the shift of $E_{g}$ due to the Fröhlich interaction.

Figure 2(a) shows the $T$ dependence of $E_{g}$ in $\mathrm{KTaO}_{3}$ which is also a quantum-paraelectric material with a soft TO phonon mode. ${ }^{40}$ The corresponding spectra of the dielectric function and of its second derivative are shown in Appendix E. Similar to STO, the value of $E_{g}$, as derived from the optical spectra, exhibits a strong increase with $T$ up to about $300 \mathrm{~K}$. The open squares show that the Fröhlich interaction accounts once more well for the $T$-dependent renormalization of $E_{g}$ below $250 \mathrm{~K}$. The determination of the $T$ dependence of $\omega_{\mathrm{TO}}$ and of $\varepsilon_{\infty}$ is once more described in the Appendixes B and D, respectively. The value of $\omega_{\mathrm{LO}}=833 \mathrm{~cm}^{-1}$ has been taken from Ref. 41. The fit using Eq. (2) yields a vertical offset of $E_{g}(T=0 \mathrm{~K})=4.87 \pm 0.01 \mathrm{eV}, A=2.24 \pm 0.06 \mathrm{eV}$, and $C=2.92 \pm 0.07$. The corresponding data and fits for $\mathrm{CaTiO}_{3}$ with $C=1.89 \pm 0.08, E_{g}(T=0 \mathrm{~K})=4.35 \pm 0.03 \mathrm{eV}$, and $A=3.30 \pm 0.15 \mathrm{eV}$ are displayed in Fig. 2(b). They confirm that the soft-mode behavior and the resulting divergence of $\varepsilon_{0}$ towards low $T$ are governing the renormalization of $E_{g}$. This confirms the important role of the Fröhlich electron-phonon interaction in the anomalous $T$ dependence of $E_{g}$ in these quantum-paraelectric materials.

In return, the characteristic $T$ dependence of $E_{g}$ can be used to monitor the soft-mode behavior and to identify a possible FE transition in these materials. We demonstrate this first for the case of oxygen isotope substituted $\mathrm{SrTi}^{18} \mathrm{O}_{3}$ for which a weak FE order that is stabilized by the reduction of the lattice quantum fluctuations develops below $T_{\text {Curie }} \approx 26 \mathrm{~K} .{ }^{42}$ The measured ellipsometry spectra and their second derivates are shown in Figs. 8(a) and 8(b) in Appendix E. Figure 2(c) shows that the $T$ dependence of $E_{g}$ exhibits indeed a weak, yet clearly noticeable, anomaly at $T_{\text {Curie }} \approx 26 \mathrm{~K}$. This anomaly is marked by an arrow and is more clearly shown in the inset which compares the low- $T$ behavior of $E_{g}$ in $\mathrm{SrTi}^{18} \mathrm{O}_{3}$ (full circles) with the one of the rescaled $E_{g}$ (by a factor of 1.0015) of $\mathrm{SrTi}^{16} \mathrm{O}_{3}$ (stars). Shown by the open squares is the contribution of the Fröhlich interaction as obtained using Eq. (2) with $C=1.88 \pm 0.05$ and $\omega_{\mathrm{LO}}=765 \mathrm{~cm}^{-1}, \varepsilon_{\infty}=$ 5.1 , and the $T$ dependence of $\omega_{\mathrm{TO}}$ as measured with infrared spectroscopy (see Appendixes B-D). The latter exhibits an anomalous hardening below $T_{\text {Curie }}$ as is shown in Fig. 4(c) in Appendix B. The fit yields $E_{g}(T=0 \mathrm{~K})=4.34 \pm 0.02 \mathrm{eV}$ and $A=3.16 \pm 0.10 \mathrm{eV}$ and reproduces the $T$ dependence of $E_{g}$ and also the anomaly around $T_{\text {Curie }} \approx 26 \mathrm{~K}$ rather well.

It has been predicted that the static displacement $u$ that occurs below the FE transition will also contribute to the hardening of $E_{g} \cdot{ }^{43}$ It reduces the symmetry of the lattice and thereby can introduce a mixing of the oxygen and titanium related bands that leads to a repulsion of the valence and conduction bands which increases $E_{g}$. Nevertheless, the good agreement in Fig. 2(c) between the measured $E_{g}$ values and the estimated contribution of the Fröhlich interaction suggests that the latter accounts for a sizable and possibly even the major part of the hardening of $E_{g}$ in the FE state.

In Fig. 2(d), we show that similar arguments likely even apply for the case of $\mathrm{BaTiO}_{3}$ for which the formation of a FE state with a large polarization below $T_{\text {Curie }}=405 \mathrm{~K}$ gives rise to a much stronger anomaly in the $T$ dependence of $E_{g}$ than in $\mathrm{SrTi}^{18} \mathrm{O}_{3}$. Figure 2(d) shows that whereas $E_{g}$ is only weakly $T$ dependent in the paraelectric state at $T>T_{\text {Curie }}$, right below $T_{\text {Curie }}$ it exhibits a steep increase that is about 50 times stronger than the one in $\mathrm{SrTi}^{18} \mathrm{O}_{3}$. In $\mathrm{BaTiO}_{3}$ the situation is somewhat complicated by the fact that there exist two soft TO modes that both contribute to the divergence of $\varepsilon_{0}$ at $T_{\text {Curie }} \cdot{ }^{44,45}$ The additional low-frequency mode (the so-called central mode) accounts here for the order-disorder component of the FE transition which apparently is of a mixed character. Accordingly, we explicitly included the $T$ dependence of both soft TO modes in the Lyddane-Sachs-Teller relation using $\omega_{\mathrm{TO}}$ as reported in Refs. 44-46. The values of $C=$ $4.78 \pm 0.20, \omega_{\mathrm{LO}}(300 \mathrm{~K})=716 \mathrm{~cm}^{-1}$, and $\varepsilon_{\infty}=5.3$ were once more obtained from our IR ellipsometry data as described in Appendixes $\mathrm{C}$ and $\mathrm{D}$, respectively. The open squares in Fig. 2(d) show that the large anomaly of $E_{g}$ in $\mathrm{BaTiO}_{3}$ can be reasonably well reproduced in terms of the Fröhlich interaction with the fit parameters $E_{g}(T=0 \mathrm{~K})=3.72 \pm 0.01 \mathrm{eV}$ and $A=0.63 \pm 0.01 \mathrm{eV}$ that are not too far from the ones in STO. We notice that the agreement could be further improved by including additional contributions from the coupling to the other phonon modes (which do not soften toward $T_{\text {Curie }}$ ) and the thermal lattice expansion. These can become rather sizable in the vicinity of $T_{\text {Curie }}=405 \mathrm{~K}$ and may, for example, account for the rather weak $T$ dependence of $E_{g}$ in the paraelectric state.

Last but not least, we show in Fig. 3 that the anomaly in the $T$ dependence of $E_{g}$ can be used to identify the FE order in STO thin films. It is well known that a FE order can be induced in STO thin films that are grown on substrates which introduce a strong tensile strain. ${ }^{5}$ For orthorhombic, (110)-oriented $\mathrm{DyScO}_{3}$ substrates, the average mismatch with respect to the lattice parameters of bulk $\mathrm{SrTiO}_{3}$ is about $+1 \%$. 

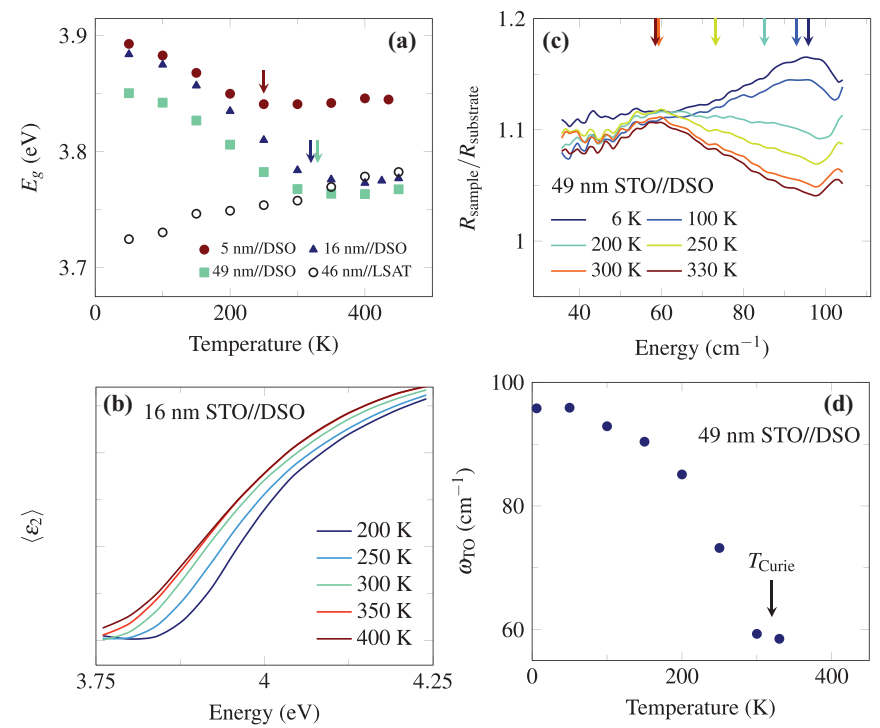

FIG. 3. (Color online) (a) $T$ dependence of $E_{g}$ in strained STO thin films on $\mathrm{DyScO}_{3}$ and LSAT substrates. Arrows mark the anomalies at $T_{\text {Curie }}$ for the films on $\mathrm{DyScO}_{3}$. (b) Evolution of the as-measured pseudodielectric function $\left\langle\varepsilon_{2}\right\rangle$ of the 16-nm STO film on $\mathrm{DyScO}_{3}$. (c) Ratio of the FIR reflectivity of sample and bare substrate for the 49-nm-thick STO film on $\mathrm{DyScO}_{3}$. The arrows indicate the soft-mode frequency $\omega_{\text {TO }}$ for which the $T$ dependence is detailed in $(\mathrm{d})$.

For fully lattice-matched STO, this amounts to a sizable tensile stress of $\sim 1 \mathrm{GPa}$ and an induced FE transition at $T_{\text {Curie }} \sim$ $300 \mathrm{~K}$. This FE state has been previously identified with piezoforce scanning microscopy, ${ }^{8}$ synchrotron x-ray diffraction, ${ }^{9}$ and more recently with $\mathrm{THz}$ and FIR spectroscopy studies of the $T$ dependence of the soft TO mode of STO. ${ }^{47}$

Figure 3(a) shows that the strain-induced FE order in these STO thin films can be also conveniently identified with optical spectroscopy. The solid symbols display the $T$ dependence of $E_{g}$ for a series of STO films on $\mathrm{DyScO}_{3}$ substrates with thicknesses of 49,16 , and $5 \mathrm{~nm}$, respectively. The characteristic anomaly, as indicated by the arrows, serves as a clear fingerprint of the FE transition at $T_{\text {Curie }} \sim 330,320$, and $250 \mathrm{~K}$, respectively. For the case of the 49-nm-thick STO film, we have confirmed the FE transition at $T_{\text {Curie }}=330 \mathrm{~K}$ with far-infrared reflectivity measurements which directly probe the $T$ dependence of the soft TO mode as shown in Figs. 3(c) and $3(\mathrm{~d})$. For the thinner films, these far-infrared data are increasingly difficult to interpret since the signal from the STO phonons becomes much weaker than the one from the $\mathrm{DyScO}_{3}$ substrate (which also exhibits a considerable $T$ dependence). On the other hand, Fig. 3(b) shows for the 16-nm-thick STO film that the FE transition can be readily identified in the as-measured spectra of the pseudodielectric function $\left\langle\varepsilon_{2}\right\rangle$ due to the blue-shift of the corresponding interband transition below $T_{\text {Curie }} \sim 320 \mathrm{~K}$. The enhanced sensitivity of the optical spectra to the properties of such thin films is owed to the penetration depth of the light which is much shorter in the VIS-UV range $(\sim 100 \mathrm{~nm})$ than in the FIR range $(>1000 \mathrm{~nm})$. Finally, Fig. 3(a) also shows our optical data of a STO thin film grown on a LSAT substrate which exerts a weak compressive strain that does not induce a FE transition. ${ }^{5}$ Here, the $T$ dependence of $E_{g}$ exhibits indeed no sign of an anomaly and is similar to the one in bulk STO.

\section{CONCLUSIONS}

In summary, we have shown that the anomalous $T$ dependent shift of the direct band gap of STO is strongly affected and likely even dominated by the Fröhlich electronphonon interaction with the so-called soft mode that is at the heart of its quantum-paraelectric properties. We have also demonstrated that the optical band-gap measurements can therefore be used as a very sensitive and efficient tool to search for a ferroelectric order in related bulk and thin-film materials. In particular, we have shown that the strain-induced ferroelectric order in STO films on a $\mathrm{DyScO}_{3}$ substrate can be readily detected even in films that are only $5 \mathrm{~nm}$ thick. This well-established optical technique, which is accessible in many laboratories, could be rather useful to search for ferroelectric transitions in other bulk and thin-film materials, in particular, in complex heterostructures and devices with buried ferroelectric layers. It will also be interesting to study relaxor ferroelectric, improper ferroelectric, or even multiferroic materials to investigate whether they exhibit a similar relationship between the enhancement of the dielectric constant and the renormalization of the direct band gap due to the Fröhlich-type electron-phonon interaction.

\section{ACKNOWLEDGMENTS}

This work has been supported by the Schweizer Nationalfonds (SNF) Grant No. 200020-140225 and the project "CEITEC-Central European Institute of Technology" (Project No. CZ.1.05/1.1.00/02.0068). We thank K. Conder for his help in preparing the isotope exchanged $\mathrm{SrTi}^{18} \mathrm{O}_{3}$ crystal and B. Doggett for his contribution to the PLD thin-film growth. We acknowledge stimulating discussions with M. Cardona and E. Kotomin.

\section{APPENDIX A: FITTING OF THE DIRECT INTERBAND TRANSITION}

The numerical derivatives of the real and imaginary parts $\varepsilon_{1}$ and $\varepsilon_{2}$ of the measured dielectric function have been independently calculated using an implementation of the Savitzky-Golay smoothing algorithm in PYTHON. ${ }^{48}$ The code yields also the $n$th numerical derivative of the input data with respect to the input $x$ value, i.e., the energy in our case. Care has been taken that no artificial features are introduced by the smoothing procedure. The real and imaginary parts $\frac{\partial^{2} \varepsilon_{1}}{\partial E^{2}}$ and $\frac{\partial^{2} \varepsilon_{2}}{\partial E^{2}}$ have been fitted simultaneously in the vicinity of the $M_{0}$ critical point using the second derivative of Eq. (1). After the fitting, the parameters for the energy $E$, the prefactor $S$, and the broadening $\Gamma$ for the transition have been obtained as exemplary shown in Fig. 1 for $\mathrm{SrTiO}_{3}$.

It is important to note that the maxima of the derivative spectra do not necessarily coincide with the extracted energies of the interband transitions. The phase factor $p=\frac{3 \pi}{2} \mathrm{i}$ in the exponent results in a mixing of real and imaginary parts of the dielectric function close to the resonance. This can lead to 
a small shift between the transition energy and the observed maximum in the derivative spectrum.

\section{APPENDIX B: T DEPENDENCE OF THE SOFT TO MODE}

The eigenfrequency of the transverse optical branch of the soft mode $\omega_{\mathrm{TO}}$ of $\mathrm{SrTiO}_{3}$ is known to exhibit a strong $T$ dependence that originates from the quantum-paraelectric behavior. ${ }^{37,49,50}$ Figure 4(a) shows the result of a Lorentz oscillator fit of the soft mode in the far-infrared ellipsometry spectra which has been performed over a broad temperature range. Figure 4(b) shows that a similar softening of $\omega_{\mathrm{TO}}$ is observed in the incipient-ferroelectric perovskite $\mathrm{KTaO}_{3}$ and also in $\mathrm{CaTiO}_{3}$.

In the ferroelectric state, the soft mode is expected to harden again. ${ }^{51}$ Such a behavior is indeed observed in the ferroelectric state of $\mathrm{SrTi}^{18} \mathrm{O}_{3}$ at $T \leqslant T_{\text {Curie }}=26 \mathrm{~K}$. Figure 4(c) displays the $T$ dependence of $\omega_{\text {TO }}$ which has been deduced from the peak position of the soft mode in the dielectric function. The latter has been obtained from far-infrared reflectivity data of $\mathrm{SrTi}^{18} \mathrm{O}_{3}$ by using a Kramers-Kronig transformation. It shows that the soft-mode eigenfrequency $\omega_{\text {TO }}$ exhibits a weak, yet clear minimum at $T_{\text {Curie }}=26 \mathrm{~K}$ as marked by the arrow in Fig. 4(c). In the ferroelectric phase between 30 and $5 \mathrm{~K}$ there is indeed a characteristic hardening of $\omega_{\mathrm{TO}}$ by $\sim 5 \mathrm{~cm}^{-1}$. A similar
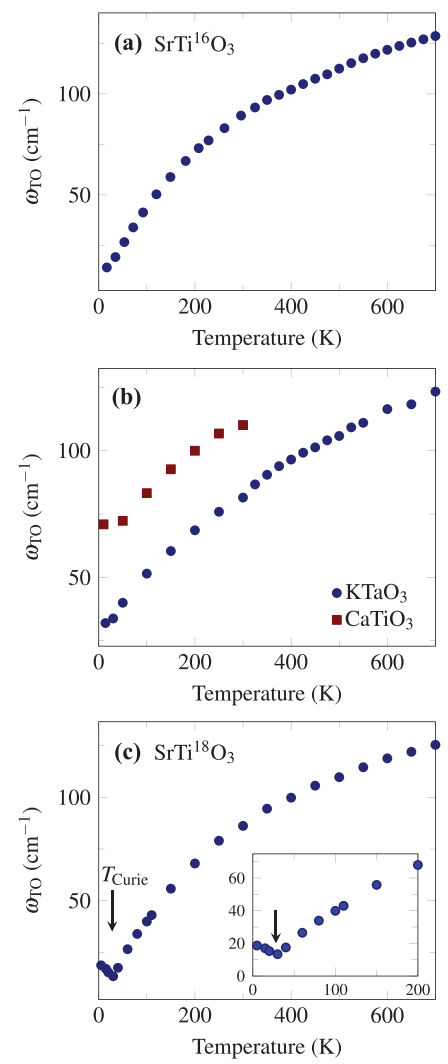

FIG. 4. (Color online) Soft-mode eigenfrequency $\omega_{\text {TO }}$ as a function of temperature as obtained from the far-infrared ellipsometry data of the quantum-paraelectric materials (a) $\mathrm{SrTiO}_{3}$ and (b) $\mathrm{KTaO}_{3}$ and polycrystalline $\mathrm{CaTiO}_{3}$. (c) Corresponding data for $\mathrm{SrTi}^{18} \mathrm{O}_{3}$ where the ferroelectric transition at $T_{\text {Curie }}=26 \mathrm{~K}$ is accompanied by a hardening of the soft mode.
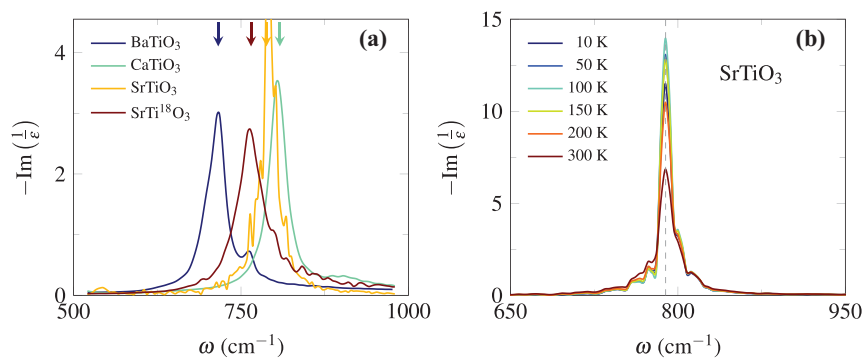

FIG. 5. (Color online) (a) Room-temperature loss function of $\mathrm{BaTiO}_{3}, \mathrm{CaTiO}_{3}, \mathrm{SrTiO}_{3}$, and $\mathrm{SrTi}^{18} \mathrm{O}_{3}$ as obtained from the dielectric functions that were measured with mid-infrared ellipsometry. The arrows indicate the LO eigenfrequencies given in the text. (b) Temperature dependence of the loss function of $\mathrm{SrTiO}_{3}$ as determined from the ellipsometry measurements at different temperatures. The dashed line marks the position of the LO mode energy of $\omega_{\mathrm{LO}}=788 \mathrm{~cm}^{-1}$.

value has been previously obtained from Raman measurements on $\mathrm{SrTi}^{18} \mathrm{O}_{3} .{ }^{52}$

The $T$ dependence of the soft mode in $\mathrm{BaTiO}_{3}$ has been extracted from the literature data for the hyper-Raman scattering, ${ }^{46}$ the far-infrared spectroscopy in Ref. 44 for $T<T_{\text {Curie }}$, and the lower-energy phonon branch as observed in the infrared spectroscopy for $T>T_{\text {Curie }}$ in Ref. 45. The estimated phase transition temperature of $T_{\text {Curie }}=405 \mathrm{~K}$ of these assembled experimental results agrees well with $T_{\text {Curie }}$ of the sample that has been investigated in this work.

\section{APPENDIX C: DETERMINATION OF $\omega_{\text {LO }}$}

In an insulator such as $\mathrm{SrTiO}_{3}$, the eigenfrequency of a longitudinal optical phonon mode $\omega_{\text {LO }}$ can be accurately determined from the zero crossing of the real part of the dielectric function $\varepsilon_{1}$ or likewise from a maximum in the
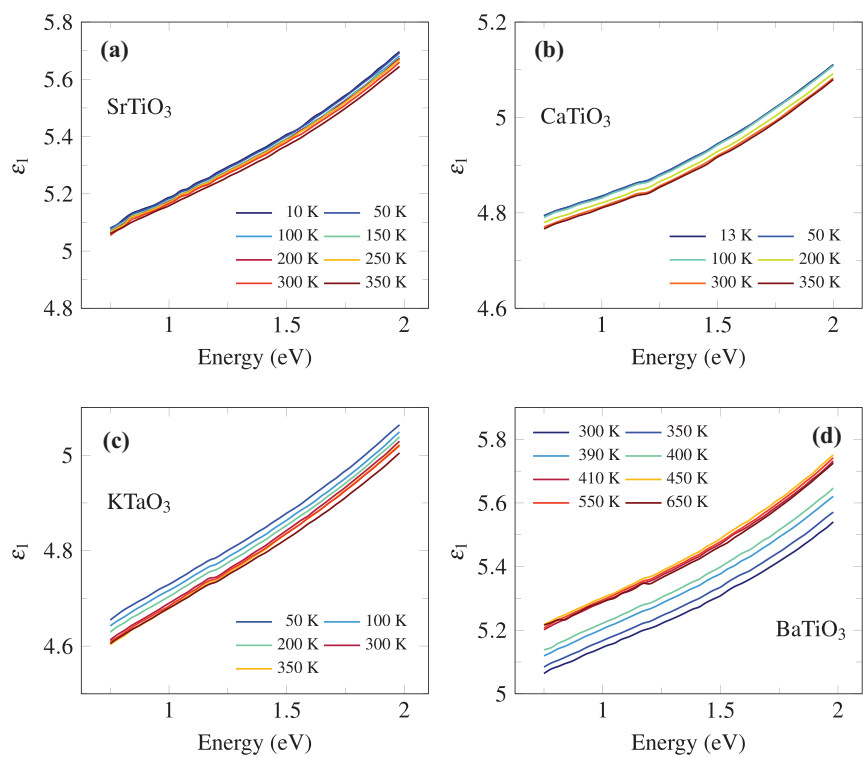

FIG. 6. (Color online) Temperature dependence of the real part of the dielectric function $\varepsilon_{1}$ as measured by ellipsometry for (a), $\mathrm{SrTiO}_{3}$, (b), $\mathrm{CaTiO}_{3}$, (c) $\mathrm{KTaO}_{3}$, and (d) $\mathrm{BaTiO}_{3}$. 

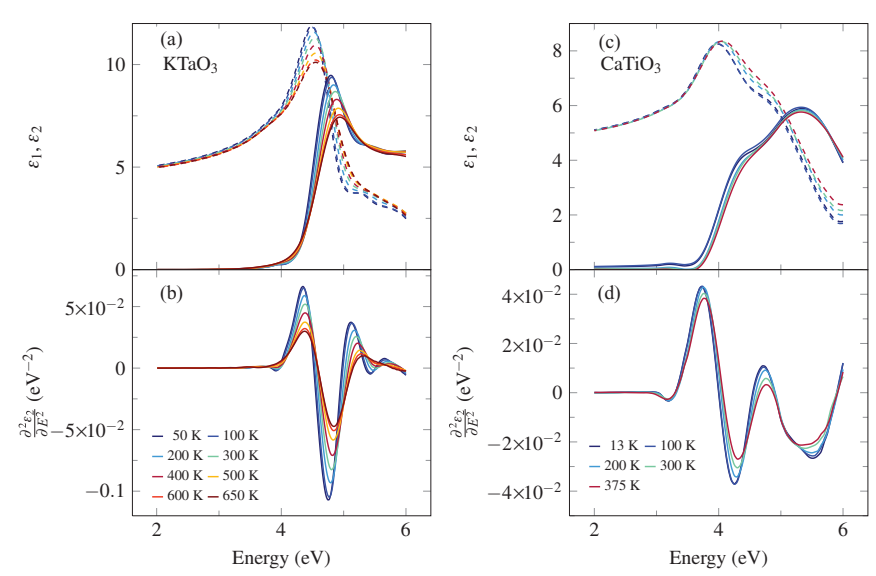

FIG. 7. (Color online) Ellipsometry spectra measured at different temperatures and the corresponding second derivative spectra for $\mathrm{KTaO}_{3}\left[(\mathrm{a})\right.$ and (b)] and $\mathrm{CaTiO}_{3}[(\mathrm{c})$ and (d)] samples.

so-called loss function

$$
-\operatorname{Im}\left(\frac{1}{\varepsilon}\right)=\frac{\varepsilon_{2}}{\varepsilon_{1}^{2}+\varepsilon_{2}^{2}} .
$$

Figure 5(a) shows for the case of the room-temperature spectra of $\mathrm{SrTiO}_{3}, \mathrm{BaTiO}_{3}, \mathrm{CaTiO}_{3}$, and $\mathrm{SrTi}^{18} \mathrm{O}_{3}$ that this procedure can be used to accurately determine the value of $\omega_{\mathrm{LO}}$ (as indicated by the arrows). For these perovskites, a large TO-LO splitting is observed due to the high ionicity of these materials. Hence, for the soft mode $\omega_{\mathrm{LO}}$ is located in the mid-infrared range at 716, 808, 788, and $765 \mathrm{~cm}^{-1}$ for $\mathrm{BaTiO}_{3}, \mathrm{CaTiO}_{3}$, $\mathrm{SrTiO}_{3}$, and $\mathrm{SrTi}^{18} \mathrm{O}_{3}$, respectively. For $\mathrm{KTaO}_{3}$ we used the value of $\omega_{\mathrm{LO}}=833 \mathrm{~cm}^{-1}$ that is reported in Ref. 41 .

In these insulating titantes, the value of $\omega_{\mathrm{LO}}$ of the soft mode is hardly temperature dependent. This is demonstrated in Fig. 5(b) for the case of $\mathrm{SrTiO}_{3}$ which shows that the position of the peak in the loss function, as derived from our mid-infrared ellipsometry measurements, hardly changes with temperature. This has been also previously shown by Servoin and Gervais in Ref. 38.

\section{APPENDIX D: DETERMINATION OF $\varepsilon_{0}$ AND $\varepsilon_{\infty}$}

We have derived $\varepsilon_{0}$ from the Lyddane-Sachs-Teller relation $\frac{\varepsilon_{0}}{\varepsilon_{\infty}}=C \frac{\omega_{\mathrm{LO}}^{2}}{\omega_{\mathrm{TO}}^{2}}$, using the values of $\omega_{\mathrm{TO}}, \omega_{\mathrm{LO}}, \varepsilon_{\infty}$, and $C$ as determined from the far- to near-infrared ellipsometry spectra. We have already shown how $\omega_{\text {TO }}$ and $\omega_{\text {LO }}$ have been obtained from these optical spectra. In the following, we discuss how the values of $C$ and $\varepsilon_{\infty}$ have been obtained from these ellipsometry data. The prefactor $C$ accounts for the $T$-independent contribution due to the other infrared-active phonon modes. The spectral weight of these modes has been
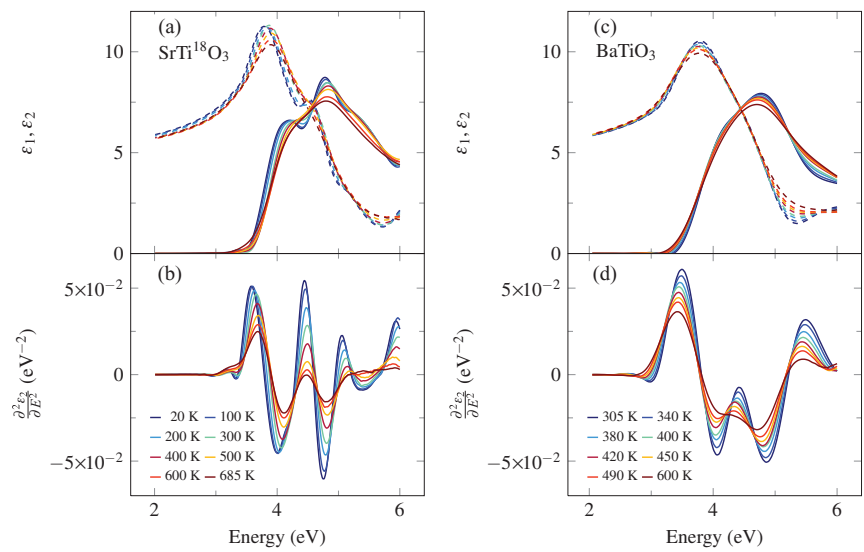

FIG. 8. (Color online) Ellipsometry spectra measured at different temperatures and the corresponding second derivative spectra for $\mathrm{SrTi}^{18} \mathrm{O}_{3}\left[(\mathrm{a})\right.$ and (b)] and $\mathrm{BaTiO}_{3}[(\mathrm{c})$ and (d)] single crystals.

obtained from the far-infrared ellipsometry data by fitting with Lorentzian functions (not shown here). The value of $\varepsilon_{\infty}$ has been deduced from the near-infrared ellipsometry data. Figure 6 shows that, for this energy range, which is well above the phonons and well below the interband transitions (or the gap energy), the real part of the dielectric function is only weakly $T$ dependent and exhibits a very moderate and continuous dispersion. For our calculations we used the value of $\varepsilon_{\infty}$ at $E=0.9 \mathrm{eV}$ at $300 \mathrm{~K}$. We note that the very weak $T$ variation of $\varepsilon_{\infty}$ of less than a percent does not strongly influence our estimate of the band-gap shift due to the Fröhlich interaction. According to Eq. (1), the Fröhlich interaction is governed by the very large $T$-dependent changes of $\varepsilon_{0}$ which arise from the soft-mode behavior.

\section{APPENDIX E: NIR-UV ELLIPSOMETRY SPECTRA $\mathrm{OF} \mathrm{KTaO}_{3}, \mathrm{CaTiO}_{3}, \mathrm{SrTi}^{18} \mathrm{O}_{3}, \mathrm{AND} \mathrm{BaTiO}_{3}$}

In Fig. 7, we show typical ellipsometry spectra for the incipient ferroelectric material $\mathrm{KTaO}_{3}$ and for $\mathrm{CaTiO}_{3}$ in terms of the real and imaginary parts of the dielectric function $\varepsilon=$ $\varepsilon_{1}+\mathrm{i} \varepsilon_{2}$ together with the corresponding second derivative spectra of the imaginary part $\frac{\partial^{2} \varepsilon_{2}}{\partial E^{2}}$. Corresponding data for the ferroelectric materials $\mathrm{SrTi}^{18} \mathrm{O}_{3}$ and $\mathrm{BaTiO}_{3}$ are shown in Fig. 8. All presented spectra have been corrected for surface roughness effects using the WOOLLAM VASE software. ${ }^{15}$ The surface roughness has been found to be on the order of $\leqslant 4 \mathrm{~nm}$ for all investigated samples and it showed only a weak variation with temperature during a measurement cycle. Only during the measurements at $T>550 \mathrm{~K}$ we have sometimes observed a decrease in the roughness that occurred during or after the measurements.
*Present address: University of Potsdam, Institute of Physics and Astronomy, Karl-Liebknecht-Strasse 24-25, D-14476 Potsdam, Germany.

${ }^{\dagger}$ christian.bernhard@unifr.ch

${ }^{1}$ Physics of Ferroelectrics-A Modern Perspective, edited by K. M. Rabe, C. H. Ahn, and J.-M. Triscone (Springer, Berlin, 2007).
${ }^{2}$ N. Setter, D. Damjanovic, L. Eng, G. Fox, S. Gevorgian, S. Hong, A. Kingon, H. Kohlstedt, N. Y. Park, G. B. Stephenson, I. Stolitchnov, A. K. Tagantsev, D. V. Taylor, T. Yamada, and S. Streiffer, J. Appl. Phys. 100, 051606 (2006).

${ }^{3}$ M. Bibes, J. E. Villegas, and A. Barthélémy, Adv. Phys. 60, 5 (2011). 
${ }^{4}$ N. A. Pertsev, A. K. Tagantsev, and N. Setter, Phys. Rev. B 61, R825 (2000).

${ }^{5}$ J. H. Haeni, P. Irvin, W. Chang, R. Uecker, P. Reiche, Y. L. Li, S. Choudhury, W. Tian, M. E. Hawley, B. Craigo, A. K. Tagantsev, X. Q. Pan, S. K. Streiffer, L. Q. Chen, S. W. Kirchoefer, J. Levy, and D. G. Schlom, Nature (London) 430, 758 (2004).

${ }^{6}$ P. Lunkenheimer, V. Bobnar, A. V. Pronin, A. I. Ritus, A. A. Volkov, and A. Loidl, Phys. Rev. B 66, 052105 (2002).

${ }^{7}$ E. Langenberg, I. Fina, J. Ventura, B. Noheda, M. Varela, and J. Fontcuberta, Phys. Rev. B 86, 085108 (2012).

${ }^{8}$ in Scanning Probe Microscopy: Electrical and Electrochemical Phenomena at the Nanoscale, edited by S. Kalinin and A. Gruverman (Springer, New York, 2006).

${ }^{9}$ S. K. Streiffer, J. M. Eastman, D. D. Fong, C. Thompson, A. Munkholm, M. V. Ramana Murty, O. Auciello, G. R. Bai, and G. B. Stephenson, Phys. Rev. Lett. 89, 067601 (2002).

${ }^{10}$ D. D. Fong, G. B. Stephenson, S. K. Streiffer, J. A. Eastman, O. Auciello, P. H. Fuoss, and C. Thompson, Science 304, 1650 (2004).

${ }^{11}$ D. A. Tenne, A. Bruchhausen, N. D. Lanzillotti Kimura, A. Fainstein, R. S. Katiyar, A. Cantarero, A. Soukiassian, V. Vaithyanathan, J. H. Haeni, W. Tian, D. G. Schlom, K. J. Choi, D. M. Kim, C. B. Eom, H. P. Sun, X. Q. Pan, Y. L. Li, L. Q. Chen, Q. X. Jia, S. M. Nakhmanson, K. M. Rabe, and X. X. Xi, Science 313, 1614 (2006).

12 "Crystec", www.crystec.de.

13“SurfaceNet”, http://www.surfacenet.de.

${ }^{14}$ R. Wang and M. Itoh, Phys. Rev. B 64, 174104 (2001).

15 “JA Woollam Co., Inc.” http://www.jawoollam.com.

${ }^{16}$ C. Bernhard, J. Humlíček, and B. Keimer, Thin Solid Films 455456, 143 (2004)..

${ }^{17}$ K. W. Kim, M. Rössle, A. Dubroka, V. K. Malik, T. Wolf, and C. Bernhard, Phys. Rev. B 81, 214508 (2010).

${ }^{18}$ M. Cardona, Phys. Rev. 140, A651 (1965).

${ }^{19}$ K. van Benthem, C. Elsaesser, and R. H. French, J. Appl. Phys. 90, 6156 (2001).

${ }^{20}$ A. Savitzky and M. J. E. Golay, Anal. Chem. 36, 1627 (1964).

${ }^{21} \mathrm{P}$. Y. Yu and M. Cardona, Fundamentals of Semiconductors, 3rd ed. (Springer, Berlin, 2005).

${ }^{22}$ P. Lautenschlager, M. Garriga, S. Logothetidis, and M. Cardona, Phys. Rev. B 35, 9174 (1987).

${ }^{23}$ V. Trepakov, A. Dejneka, P. Markovin, A. Lynnyk, and L. Jastrabik, New J. Phys. 11, 083024 (2009).

${ }^{24}$ P. Lautenschlager, P. B. Allen, and M. Cardona, Phys. Rev. B 31, 2163 (1985).

${ }^{25}$ D. Olguín, M. Cardona, and A. Cantarero, Solid State Commun. 122, 575 (2002).
${ }^{26}$ C. Keffer, T. M. Hayes, and A. Bienenstock, Phys. Rev. Lett. 21, 1676 (1968).

${ }^{27}$ P. B. Allen and M. Cardona, Phys. Rev. B 23, 1495 (1981).

${ }^{28}$ P. B. Allen and M. Cardona, Phys. Rev. B 27, 4760 (1983).

${ }^{29}$ M. Cardona, Solid State Commun. 133, 3 (2005).

${ }^{30}$ J. Bhosale, A. K. Ramdas, A. Burger, A. Muñoz, A. H. Romero, M. Cardona, R. Lauck, and R. K. Kremer, Phys. Rev. B 86, 195208 (2012).

${ }^{31}$ H. Y. Fan, Phys. Rev. 82, 900 (1951).

${ }^{32}$ M. Liu, T. R. Finlayson, and T. F. Smith, Phys. Rev. B 55, 3480 (1997).

${ }^{33}$ R. Loetzsch, A. Lübcke, I. Uschmann, E. Forster, V. Grosse, M. Thuerk, T. Koettig, F. Schmidl, and P. Seidel, Appl. Phys. Lett. 96, 071901 (2010).

${ }^{34}$ B. Ghebouli, M. Ghebouli, T. Chihi, M. Fatmi, S. Boucetta, and M. Reffas, Solid State Commun. 149, 2244 (2009).

${ }^{35}$ F. W. Lytle, J. Appl. Phys. 35, 2212 (1964).

${ }^{36}$ K. A. Müller and H. Burkard, Phys. Rev. B 19, 3593 (1979).

${ }^{37}$ H. Vogt, Phys. Rev. B 51, 8046 (1995).

${ }^{38}$ J. L. Servoin, Y. Luspin, and F. Gervais, Phys. Rev. B 22, 5501 (1980).

${ }^{39}$ A. A. Sirenko, C. Bernhard, A. Golnik, A. M. Clark, J. Hao, W. Si, and X. X. Xi, Nature (London) 404, 373 (2000).

${ }^{40}$ C. H. Perry and T. F. McNelly, Phys. Rev. 154, 456 (1967).

${ }^{41}$ P. A. Fleury and J. M. Worlock, Phys. Rev. 174, 613 (1968).

${ }^{42}$ M. Itoh, R. Wang, Y. Inaguma, T. Yamaguchi, Y.-J. Shan, and T. Nakamura, Phys. Rev. Lett. 82, 3540 (1999).

${ }^{43}$ R. F. Berger, C. J. Fennie, and J. B. Neaton, Phys. Rev. Lett. 107, 146804 (2011)

${ }^{44}$ J. Hlinka, T. Ostapchuk, D. Nuzhnyy, J. Petzelt, P. Kuzel, C. Kadlec, P. Vanek, I. Ponomareva, and L. Bellaiche, Phys. Rev. Lett. 101, 167402 (2008).

${ }^{45}$ I. Ponomareva, L. Bellaiche, T. Ostapchuk, J. Hlinka, and J. Petzelt, Phys. Rev. B 77, 012102 (2008).

${ }^{46}$ H. Vogt, J. A. Sanjurjo, and G. Rossbroich, Phys. Rev. B 26, 5904 (1982).

${ }^{47}$ D. Nuzhnyy, J. Petzelt, S. Kamba, P. Kuzel, C. Kadlec, V. Bovtun, M. Kempa, J. Schubert, C. M. Brooks, and D. G. Schlom, Appl. Phys. Lett. 95, 232902 (2009).

48 "SciPy", http://www.scipy.org.

${ }^{49}$ W. Cochran, Adv. Phys. 9, 387 (1960).

${ }^{50}$ A. S. Barker and M. Tinkham, Phys. Rev. 125, 1527 (1962).

${ }^{51}$ A. Yamanaka, M. Kataoka, Y. Inaba, K. Inoue, B. Hehlen, and E. Courtens, Europhys. Lett. 50, 688 (2000).

${ }^{52}$ M. Takesada, M. Itoh, and T. Yagi, Phys. Rev. Lett. 96, 227602 (2006). 